\title{
Biologically Inspired Miniature Water Strider Robot
}

\author{
Steve H. Suhr, Yun Seong Song, Sang Jun Lee, and Metin Sitti, Member, IEEE
}

\begin{abstract}
Recent biological studies on water strider insects revealed the detailed mechanism of their staying and walking on water. While macro scale bodies use buoyancy to stay on water, these very light and small insects balance their weight using repulsive surface tension forces where the insect legs are covered with hydrophobic micro-hairs. Utilizing the unique scaling advantage of these insects, this paper proposes a biologically inspired miniature micro-robot walking on water with a similar principle. The paper focuses on understanding physical characteristics of the insect and designing a robot that mimics the insect's movement. Highly hydrophobic Teflon ${ }^{\circledR}$ coated wires are used for the legs to take advantage of surface tension force, and the robot body is made of carbon fibers for minimal weight. A T-shaped actuation mechanism with three PZT-5H based unimorph actuators is utilized to move the side legs of the robot independently for controlled locomotion. Kinematics and dynamic properties of the robot prototype are analyzed and compared with the experimental results. The tethered robot can successfully move forward, backward and can also make turns. Maximum speed of the robot in forward motion is $2.3 \mathrm{~cm} / \mathrm{s}$. In the future, environmental monitoring applications on dams, lakes, sea, etc. would become possible using a network of these robots with miniature sensors, an on-board power source and electronics.
\end{abstract}

Index Terms - Micro-robotics, advanced mechanisms, biologically inspired robots, scaling effects, mobile robotics

\section{INTRODUCTION}

A DAPTING highly efficient, multi-functional, and sub-optimal biological system working principles to synthetic technologies is one of the current challenges of engineering design. Biologically inspired systems and robots can enable us to understand nature in more depth, and also provide alternative means of developing smart and advanced novel robotic mechanisms. For example, the cockroach's mobility on any terrain has been adapted to small walking robots $[1,2]$; the gecko's ability to climb on any surface

Manuscript received January 21, 2005; revised June 20, 2005.

Steve H. Suhr and Yun Seong Song are with the Department of Mechanical Engineering, Carnegie Mellon University, Pittsburgh, PA 15213 USA.

Sang Jun Lee, was with the Department of Mechanical Engineering, Carnegie Mellon University. He is now with the Department of Civil and Environmental Engineering, Carnegie Mellon University, Pittsburgh, PA 15213 USA.

Metin Sitti is with the Department of Mechanical Engineering, the Department of Electrical and Computer Engineering, and the Robotics Institute, Carnegie Mellon University, Pittsburgh, PA 15213 USA (phone: 412-268-3632; fax: 412-268-3348; e-mail: msitti@andrew.cmu.edu). vertically or upside down in very high speeds and maneuverability has been adapted to new climbing robot mechanisms [3]; and the fish's agility under water has been mimicked to improve underwater locomotion of robots [4,5]. Especially, newly emerging micro/nano-robotics area has been inspired by small biological organisms such as insects since they provide valuable insights to many less understood phenomena at the small-scale due to different physical laws and scaling effects.

Conventional macro scale locomotive systems on water rely on the buoyancy force, which is proportional to volume submerged under the surface of the water. However, when the floating object is scaled down to millimeter sizes by a ratio of $1 / \mathrm{L}$, buoyancy force decreases by $1 / \mathrm{L}^{3}$. Then, surface forces such as repulsive surface tension forces that are proportional to 1/L start to dominate the buoyancy force. Water striders use this scaling effect to stay and walk on water without breaking the water surface. Therefore, this unique locomotion mechanism on water has very little drag and enables highly maneuverable and fast motion. A water strider can go up to peak speeds of $1.5 \mathrm{~m} / \mathrm{sec}$. Recently, the unique characteristics of the water strider have been studied and understood, including the super-hydrophobicity of the legs [6] and its static and dynamic locomotion behaviors [7]. These features suggest a new mechanism that will enable miniature robots to walk on water. $\mathrm{Hu}$ et al. [7] proposed a robot similar to the insect that uses elastic energy storage to move its legs for a forward motion for a short time. This paper proposes a new micro-robot capable of walking on water inspired by a water strider that will be programmable and controllable. Similar to the biological system, this robot is miniature, power efficient and highly maneuverable, and demonstrates surface tension dominated locomotion on water. Another advantage of utilizing surface tension as the primary source of locomotion in this robot is its added mobility on and accessibility to shallow water, where boat-like designs are limited by the device displacing water underneath the surface for movements.

To understand the detailed mechanism of water strider locomotion and agility on water surface, the water strider leg and water-surface interaction were analyzed. A miniature motion mechanism integrated with unimorph piezoelectric actuators was designed and analyzed to enable desired trajectories similar to water strider legs.

The robot may be used for near future environmental monitoring, entertainment or educational applications. 


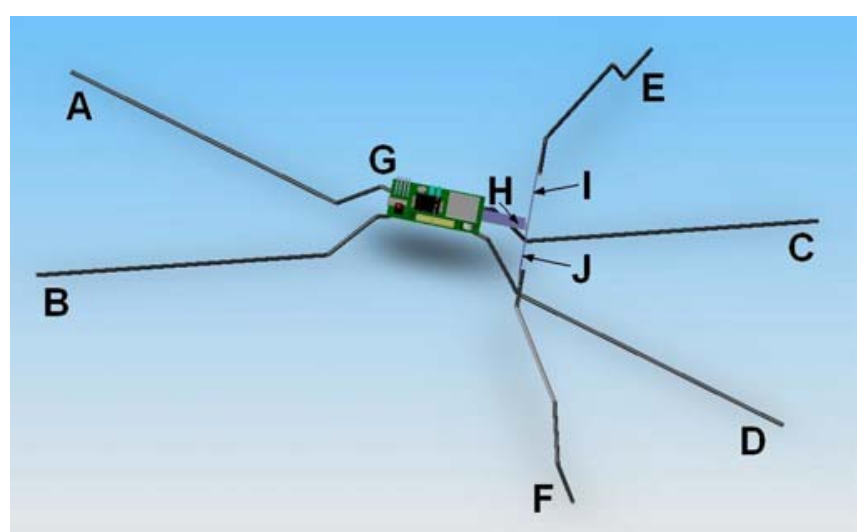

Fig. 1. Conceptual 3-D CAD model of the water strider robot: A, B, C and D are the supporting legs; $\mathrm{E}$ and $\mathrm{F}$ are the actuating legs; $\mathrm{G}$ is the body with sensors, power sources and a wireless communication module; $\mathrm{H}$ is the middle actuator; and $\mathrm{I}$ and $\mathrm{J}$ are the right/left actuators.

\section{Design OF THE RoBot}

In order to mimic the water strider, the physical characteristics of the insect were considered for robot design. There were two major features of the insect that are of high interest for designing the robot: its ability to stay on water, and the sculling motions of its middle legs that enable movement on water [7]. The whole design process was aimed for the robot to achieve similar functionality.

The conceptual model of the water strider robot is shown in Fig. 1. To reduce the total weight of the robot without compensating structural integrity, three layers of carbon fibers in different orientations are used in the body ( $G$ in Fig. 1). Ultimately, this body will include on-board electronics, power source, communication, controller, and sensors. The robot is designed to have four supporting legs (A, B, C, and D in Fig. 1) and two steering legs (E and $\mathrm{F}$ in Fig. 1). The appropriate material and shape for the supporting legs to ensure stability on water are studied in Section II.A.

Once the supporting body structure was established, actuator selection for the steering legs was the critical issue. As requirements for such a miniature robot, the steering actuators should be light, compact, fast, have large strain, have enough force output, and have low power consumption. Exploration of possible miniature actuators leads to the following conclusions: Shape Memory Alloy wires are too slow and consume excessive power; Ionic Polymer-Metal Composite actuators do not provide adequate output force and consume too much power; and conventional motors are too heavy. Therefore, piezoelectric actuators were selected for this work. They are fast, light, compact, easy to fabricate, and have low power consumption. Moreover, the simple design and fabrication method of piezoelectric unimorph actuators was most suitable for the water strider robot [8]. However, they have small motion and require high voltages. To compensate for these problems, the actuator motion is amplified by long compliant legs and resonant actuation.

Because piezoelectric actuators have only one degree of freedom, multiple actuators need to be superimposed to create complex motion. The actuators were arranged such that the resulting trajectory of the motion is expected to be elliptical to produce the sculling motion. Specifically, piezoelectric materials were attached on a T-shaped stainless steel frame as shown in Fig. 1 (H, I and J). Following sections discuss how different solution methods confirm expected motions.

\section{A. Lift Force Characterization}

The water strider maintains its body on the water surface by taking advantage of the surface tension force of the water. The legs of the insect deform the water surface without breaking it to stay on the surface. The weight of the water volume pushed down is equivalent to the lifting force the insect experiences [9]. That is, the weight of the water that would fill the volume of the dimple created from pushing the leg against the water surface is equal to the sum of the vertical components of the lifting forces. When $\boldsymbol{f}_{\boldsymbol{T}}$ is the surface tension force and $\boldsymbol{f}_{\boldsymbol{B}}$ is the buoyancy force, the effective lifting force, $F_{L}$, can be written as

$$
F_{L}=\mathbf{z} \cdot\left(\boldsymbol{f}_{\boldsymbol{T}}+\boldsymbol{f}_{\mathbf{B}}\right)=W,
$$

where $\mathbf{z}$ is the unit length vector normal to the original surface of the water and $W$ is the weight of the water that would fill the volume bounded above by the $\mathrm{z}=0$ surface and below by the water-air interface and the water-solid interface (Fig. 2).

From the Young-Laplace equation, any given point on the water-air interface profile is governed by

$$
\rho g z=\gamma\left(\frac{1}{R_{1}}+\frac{1}{R_{2}}\right),
$$

where $\rho$ is the density of water, $g$ is the gravitational constant, $z$ is the height difference of the point from the original water surface, $\gamma$ is the surface tension coefficient, and $R_{l}$ and $R_{2}$ are the principle radii of curvature of the water-air interface at the given point. Assuming the legs of the robot to be long and cylindrical $\left(R_{2}=\infty\right)$, and using boundary conditions from the contact angle information, Eq. (2) can be solved numerically to describe the shape of the water-air interface as shown in Fig. 3.

From the numerical solution, we can estimate the maximum lift force that can be generated by the synthetic legs of the water strider robot. Fig. 4 shows the numerically solved maximum lift forces for a $2 \mathrm{~cm}$ long cylinder with a radius of $0.165 \mathrm{~mm}$, the radius of the wires used in the actual robot. Experimental values of the maximum forces for plain stainless steel wire, Teflon ${ }^{\circledR}$ coated wire and Fluorothane ${ }^{\circledR}$ MP coated wire are also shown. The results suggest that if Teflon coated wires with the known contact angle of $112^{\circ}$ are used as the supporting legs, one can expect a maximum of $1.4 \mathrm{mN} / \mathrm{cm}$ or approximately $0.14 \mathrm{~g} / \mathrm{cm}$ of lift force. It can be seen from Fig. 4 that the more hydrophobic the material of the leg is, the more the maximum lift force for the robot will be. However, after a contact angle of $90^{\circ}$, maximum lift forces do not change significantly.

As a summary of supporting and steering leg design rules for staying on water without breaking the water surface are: 


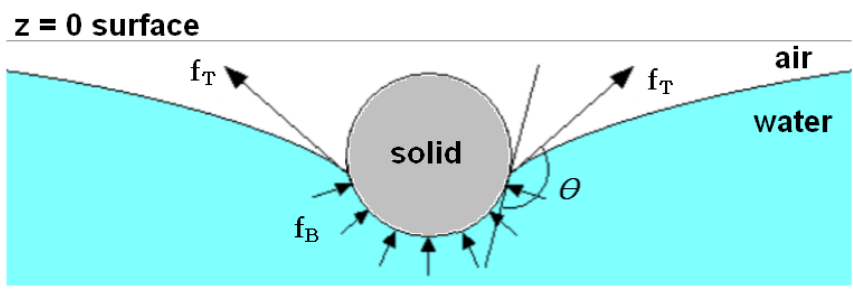

Fig. 2. Force components acting on a partially submerged solid cylinder body. $\theta$ is the contact angle between the solid body and water.

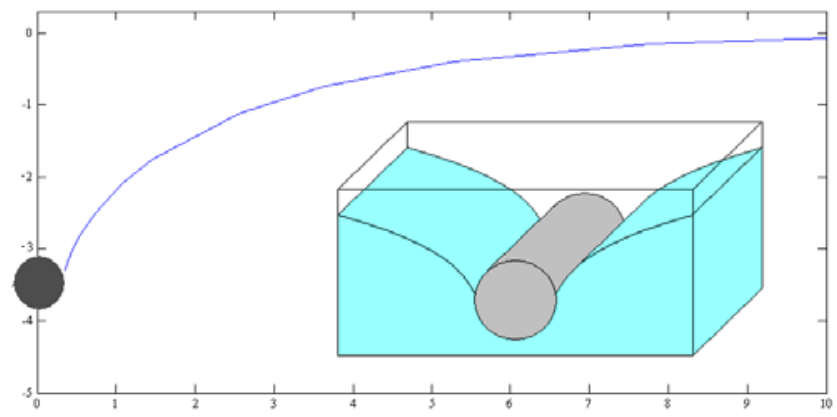

Fig. 3. Numerically solved water profile of the air-water interface. The radius of the cylinder is $0.3 \mathrm{~mm}$, and the contact angle is $112^{\circ}\left(\right.$ Teflon $\left.^{\circledR}\right)$. Scales are in $\mathrm{mm}$.

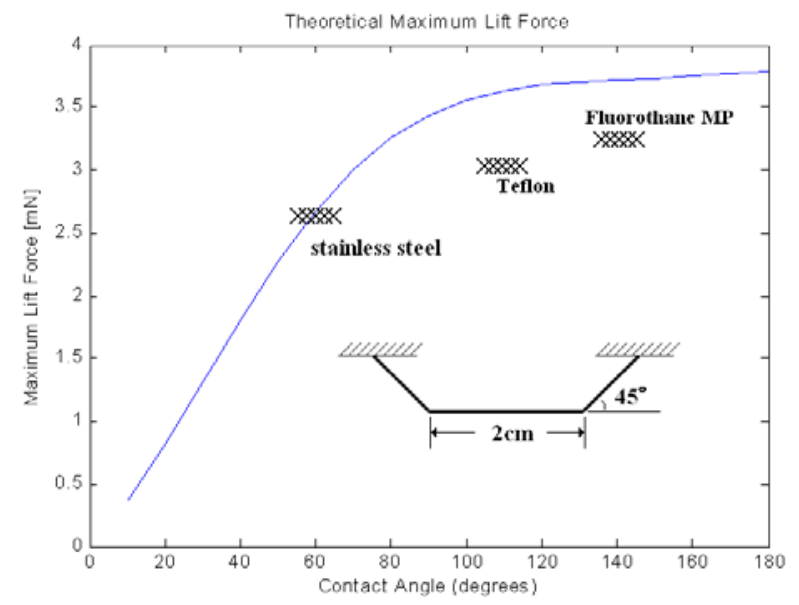

Fig. 4. Numerically solved maximum lift forces of the specimen along with three experimental data.

1) Lift force is linearly proportional to the leg length in contact with water; so, longer legs can support more weight.

2) The supporting legs should be stiff in order to not lose lift forces due to the bending of the leg.

3) Leg material contact angle should be much larger than $90^{\circ}$ for maximum lift.

4) Steering leg's normal force per given length on water surface should not exceed its maximum lift force per unit length $\left(1.4 \mathrm{mN} / \mathrm{cm}\right.$ for Teflon $\left.{ }^{\circledR}\right)$.

5) Leg end tip shape should be bent upwards slightly in order to prevent a singularity at the tip, which would decrease the lifting capability.

6) Leg radius is insignificant for the surface tension force. But smaller is better for a surface tension dominated locomotion and to minimize overall weight.
7) To maximize the lift force, the distance between the neighboring legs must exceed the width of the dimple they create.

\section{B. Piezoelectric Unimorph Actuator Analysis}

A T-shaped mechanism used in the water strider robot can be divided into three unimorph piezoelectric actuators (Fig. 5) with an extension part as shown in Fig. 6. Among various kinds of piezoelectric materials, $\mathrm{Pb}(\mathrm{Zr}, \mathrm{Ti}) \mathrm{O}_{3}$ (PZT-5H, Piezo Systems Inc.) is chosen $[8,10]$. Stainless steel is used as the elastic layer of the unimorph actuator. Kinematic analysis of three actuators involves the deflection of each unimorph actuator. Therefore, it is essential to study a single unimorph actuator.

Assuming a small deflection, the end tip deflection, $\delta$, is given by [11]

$$
\begin{aligned}
\delta & =L_{P}\left(\frac{L_{P}}{2}+L_{s}-L_{P}\right) C_{M} M_{V} V \\
& -\left[C_{M}\left(\frac{1}{3} L_{P}^{3}+L_{P}^{2}\left(L_{s}-L_{P}\right)+L_{P}\left(L_{s}-L_{P}\right)^{2}\right)+\frac{6\left(L_{s}-L_{P}\right)^{2}}{w E_{S} t_{S}^{3}}\right] f,
\end{aligned}
$$

where $V$ is applied voltage and $f$ is externally applied force. The curvature per unit length, $C_{M}$, and the torque per unit length across electrodes, $M_{V}$, are given by

$$
\begin{aligned}
& C_{M}=\frac{12}{w E_{S} t_{S} t_{P}^{2}} \frac{A B(A B+1)}{A^{2} B^{4}+2 A\left(2 B+3 B^{2}+2 B^{3}\right)+1} \\
& M_{V}=-d_{31} w E_{p} t_{p} A B(B+1) /(2 A B+2),
\end{aligned}
$$

where $A=E_{s} / E_{p}, B=t_{s} / t_{p}$. Descriptions of other variables in Eq. (3) and (4) are given in Table I.

TABLE I

PARAMETERS OF THE UNIMORPH ACTUATORS AND LEGS IN EQ. (3)-(5) AND FIG. 5-6

\begin{tabular}{ll}
\hline \hline Parameter & \multicolumn{1}{c}{ Description } \\
\hline$d_{31}$ & Piezoelectric coefficient coupling $z$ electric field to $x$ strain \\
$E_{p}$ & Elastic modulus of piezoelectric actuator \\
$E_{s}$ & Elastic modulus of stainless steel \\
$L_{1}$ & Length of the stainless steel base for the middle PZT-5H \\
& beam \\
$L_{l s}$ & Effective length of the stainless steel base for the middle \\
& PZT-5H beam \\
$L_{l p}$ & Length of the middle PZT-5H beam \\
$L_{2}$ & Length of the stainless steel base for the right or left \\
& PZT-5H beam \\
$L_{2 s}$ & Effective length of the stainless steel base for the right or \\
$L_{2 p}$ & left PZT-5H beam \\
$w$ & Length of the right or left PZT-5H beam \\
$t_{p}$ & Width of the stainless steel and PZT-5H beam \\
$t_{s}$ & Thickness of the PZT-5H actuator \\
$t_{f}$ & Thickness of stainless steel beam \\
Leg $_{1}$ & Thickness of the folded spacer \\
Leg $_{2}$ & Part 1 length of the actuating leg \\
Leg $_{3}$ & Part 2 length of the actuating leg \\
Leg $_{4}$ & Part 3 length of the actuating leg \\
\hline \hline
\end{tabular}




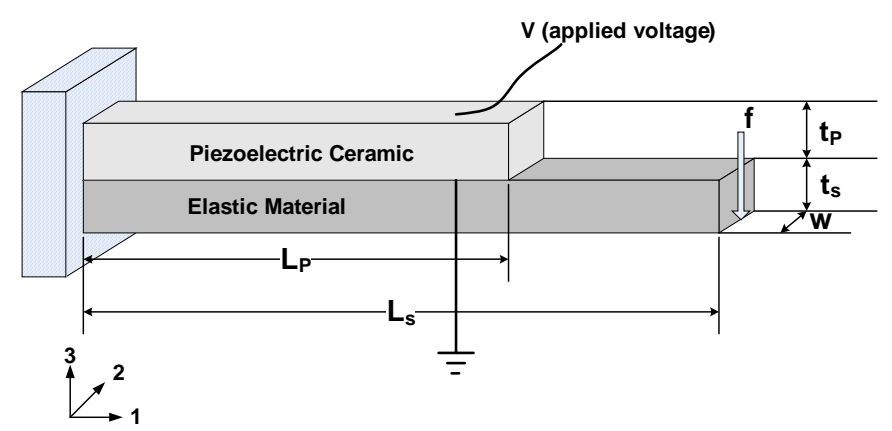

Fig. 5. Schematic of the unimorph piezoelectric actuator with the extended stainless steel layer.

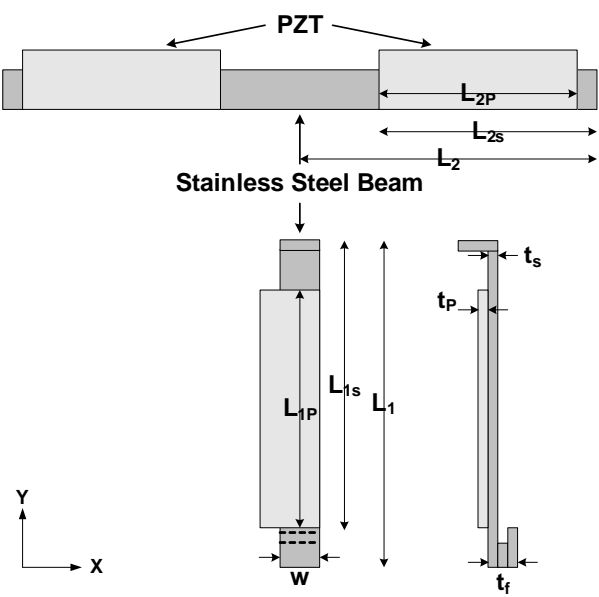

(a)

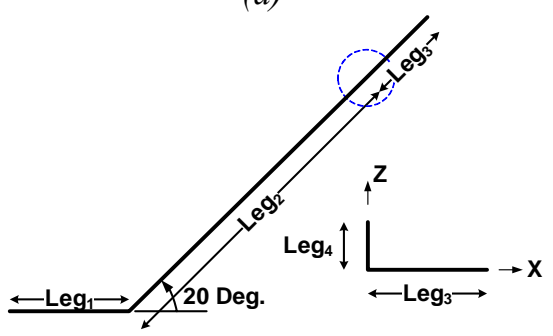

(b) $\operatorname{Leg}$

Fig. 6. Schematics of (a) the T-shape motion mechanism using three PZT-5H based unimorph actuators and (b) one of the side legs.

Because each unimorph actuator is driven by a separate signal, the three piezoelectric unimorph actuators are assumed to be basically independent and decoupled in motion. Thus, the system can be represented by using superposition of three independent unimorph actuators.

From the dimensions of each actuator and using Eq. (3), the maximum displacement of the vertical actuator, $L_{1}$, is $124.1 \mu \mathrm{m}$. The maximum displacement of the horizontal actuator, $L_{2}$, is $108.6 \mu \mathrm{m}$ because the extension parts differ in size. These results were obtained at the quasi-static state without consideration of the resonance. The modal characteristics will be analyzed in the next section.

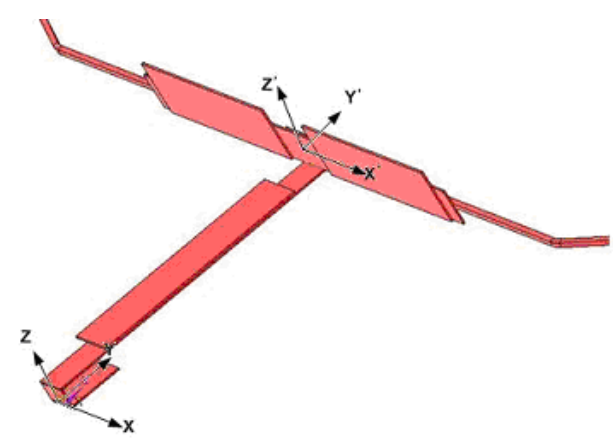

Fig. 7. Global coordinate system (XYZ) and local coordinate system (X'Y'Z').

\section{Kinematic Analysis}

The elliptical motion of the T-shaped steering mechanism is verified using the kinematic relations. Global and local coordinate systems are defined as shown in Fig. 7.

The deflection of each piezoelectric actuator is assumed to be small. From the homogeneous transformation matrix $\boldsymbol{F}$ and the local position vector of the leg's tip $\boldsymbol{p}$, its global position can be obtained as follows:

$$
\mathbf{F}=\left[\begin{array}{cccc}
1 & 0 & 0 & 0 \\
0 & \cos \alpha & -\sin \alpha & \left(L_{1}-L_{1 s}\right)+L_{1 s} \cos \alpha-\frac{w \sin \alpha}{2} \\
0 & \sin \alpha & \cos \alpha & t_{f}+L_{1 s} \sin \alpha+\frac{w \cos \alpha}{2} \\
0 & 0 & 0 & 1
\end{array}\right]
$$

$$
\mathbf{p}=\left[\begin{array}{c}
\left(L_{2}-L_{2 s}\right)+\left(L_{2 s}+L_{e g}\right) \cos \beta+\left(L_{e g}+L_{e g}\right) \cos (\beta+\zeta) \\
\left(L_{2 s}+L_{e} g_{1}\right) \sin \beta+\left(L_{e g}+L_{e g}\right) \sin (\beta+\zeta) \\
-\operatorname{Leg}_{4} \\
1
\end{array}\right],
$$

where $a \approx \arctan \left(\delta_{v} / L_{1 s}\right), \beta \approx \arctan \left(\delta_{h} / L_{2 s}\right)$ and $\zeta=20^{\circ}$ (the sweep angle of the leg). $\delta_{v}$ and $\delta_{h}$ are vertical and horizontal actuator deflections, respectively.

The global position is the product of the homogeneous transformation matrix and the local position vector:

$$
(x, y, z)^{T}{ }_{t i p}=\boldsymbol{F} \boldsymbol{p} .
$$

When the unipolar sinusoidal voltage with peak-to-peak amplitude of $150 \mathrm{~V}$ and frequency of $1 \mathrm{~Hz}$ is given and the phase difference between the horizontal and the vertical PZT actuators is $90^{\circ}$, the trajectory of the elliptic tip motion is shown in Fig. 8. At this low frequency actuation, the resulting elliptic motion has amplitude of around $0.4 \mathrm{~mm}$ in the $y$-direction and $0.2 \mathrm{~mm}$ in the $z$-direction. Table II shows the results of the static analysis. Deflection in the y-direction, parallel to the direction of forward motion of the robot, is the largest given the same amplitude of input signals to all PZT actuators, and the overall motion amplification is around 6 due to the leg kinematics. 


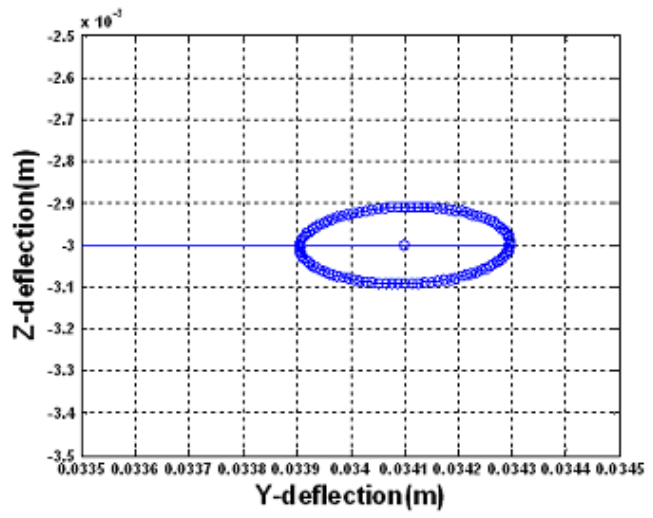

Fig. 8. Simulated trajectory of the leg's tip (magnified side view).

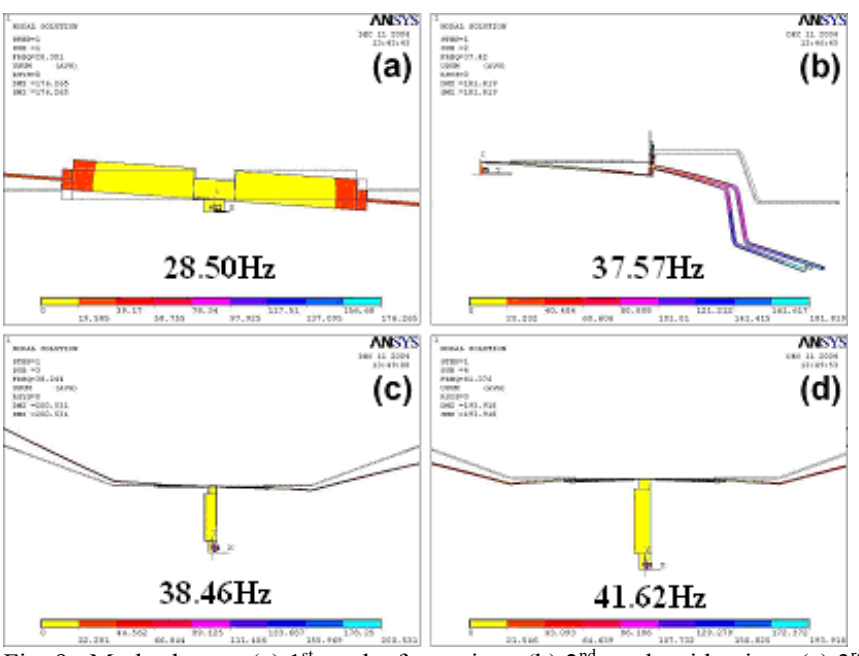

Fig. 9. Mode shapes: (a) $1^{\text {st }}$ mode, front view; (b) $2^{\text {nd }}$ mode, side view; (c) $3^{\text {rd }}$ mode, top view; (d) $4^{\text {th }}$ mode, top view.

TABLE II

DISPLACEMENT OF THE ACTUATORS AND THE LEG TIP AND ACTUATOR MOTION AMPLIFICATION FOR VARIOUS DC VOLTAGES

\begin{tabular}{cccc}
\hline \hline Voltage (V) & $\begin{array}{c}\text { Displacement of } \\
\text { Actuators (mm) }\end{array}$ & $\begin{array}{c}\text { Displacement of } \\
\text { Leg Tip (mm) }\end{array}$ & $\begin{array}{c}\text { Amplifi- } \\
\text { cation }\end{array}$ \\
\hline \multirow{2}{*}{50} & 0.056 & $\begin{array}{c}0.344 \\
(0.201)^{*}\end{array}$ & 6.143 \\
100 & 0.112 & $\begin{array}{c}0.689 \\
(0.401)^{*}\end{array}$ & 6.152 \\
& & $\begin{array}{c}1.033 \\
(0.602)^{*}\end{array}$ & 6.149 \\
\hline \hline 0*: Analytic values using MATLAB & &
\end{tabular}

\section{Finite Element Method Analysis}

Besides the motion amplification due to the steering leg kinematics, resonant actuation was also used to amplify the steering leg stroke. Therefore, a 3D finite element analysis with ANSYS $^{\circledR}$ was performed to observe resonant frequencies of the entire steering mechanism. Knowing the resonant frequencies and their mode shapes helped to design the right driving frequencies for forward/backward and turning motions. Fig. 9 shows the first four resonant mode shapes and their frequencies. Here, the $3^{\text {rd }}$ and the $4^{\text {th }}$ modes enable out-of-phase and in-phase vibration of the left and right steering actuators (I and $\mathrm{J}$ in Fig. 1) respectively. The $3^{\text {rd }}$ mode enables turning of the robot and the $4^{\text {th }}$ one does the forward/backward motion. Thus,

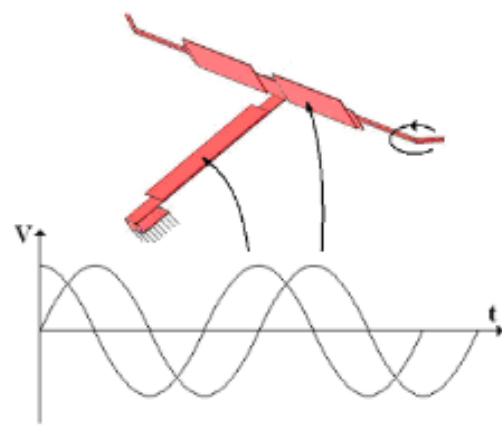

Fig. 10. Example of input signals and the resulting motion of the leg.

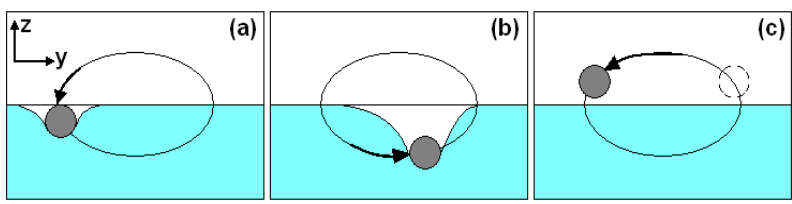

Fig. 11. Trajectory of the end tip of the steering legs.

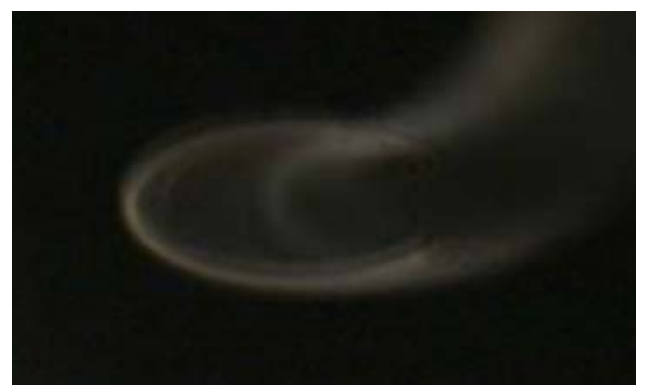

Fig. 12. Photo of the elliptical motion of the end tip of the steering legs, driven at $41 \mathrm{~Hz}$ (close to the $4^{\text {th }}$ mode) and $20 \mathrm{~V}$ peak-to-peak signals. The width of the ellipse is approximately $2 \mathrm{~mm}$.

by just changing the driving signals of all actuators to excite different modes, steering control can be realized in this mechanism. Since the quality factor of the fabricated PZT-5H based unimorph actuators is approximately 22 , resonant modes amplify the static motion of the unimorph actuators up to 130 times (including the kinematic amplification factor of 6.1 from Table II). This would drop the actuator output force at the actuating leg tip with the same amount. For example, $5 \mathrm{mN}$ of piezo-actuator force would be reduced to $0.019 \mathrm{mN} / \mathrm{cm}$ for a 2 $\mathrm{cm}$ actuating leg contacting with water, which is far less than the maximum steering leg output force of $1.4 \mathrm{mN} / \mathrm{cm}$ in Section II.A. Thus the water surface would not be broken at any time of actuation. The force output of the piezoelectric unimorph actuator can be found using Eq. (3), by setting $\delta=0$ and solving for $f$, which is the blocking force needed to keep the displacement $(\delta)$ zero.

\section{E. Locomotion Mechanism}

Motions of the three piezoelectric unimorph actuators were expected to produce elliptical motions of the actuating legs. Fig. 10 shows the signals fed in to the unimorphs and the expected leg motion simulated in the previous section.

Two sinusoidal AC voltage signals with a phase difference of $90^{\circ}$ were applied to the actuators. If the signal to the middle actuator was $90^{\circ}$ faster than the signals to the left actuator, the end tip of the left actuating leg would create counter clockwise 


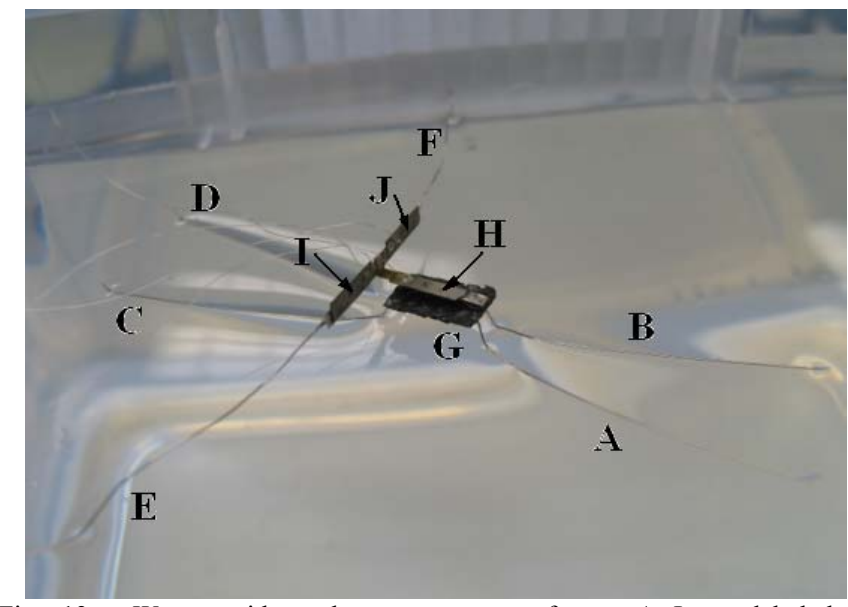

Fig. 13. Water strider robot on water surface. $\mathrm{A} \sim \mathrm{J}$ are labeled in correspondence with Fig. 1.

elliptical motion as shown in Fig. 10 and 11. The experiments validated that the end tip of the leg creates an elliptical motion as shown in Fig. 12. If the signal to the middle actuator lagged $90^{\circ}$ behind the other signal, the end tip of the leg drew a clockwise ellipse. Thus by switching the phase of the middle actuator signal, one can achieve forward/backward motion. Similarly, having two opposing sinusoidal signals to right/left actuators to excite the $3^{\text {rd }}$ mode can create two different elliptical motions at the end of the legs, one rotating clockwise while the other rotates counter clockwise. This can be used to turn the robot.

\section{EXPERIMENT}

As discussed previously, the hydrophobicity of legs is

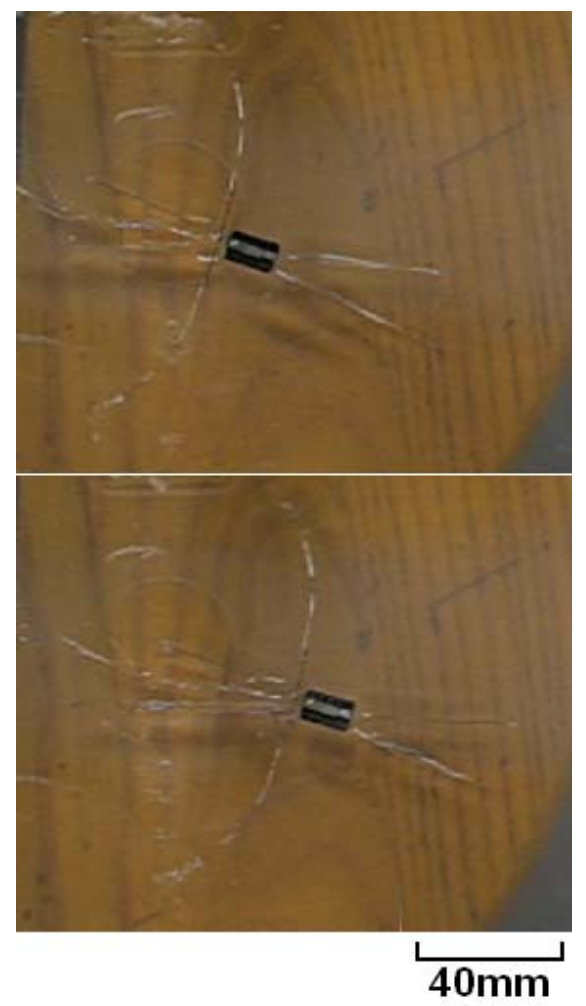

Fig. 14. Forward motion of the robot; $26 \mathrm{~mm}$ in 1.2 second interval. crucial in supporting the weight of the water strider robot. The more hydrophobic the material of the leg is, the higher the maximum lifting force one can produce. Four stainless steel legs could support up to a total mass of 1.13 grams including four legs and the carbon fiber body. Improving the contact angle with a Teflon ${ }^{\circledR}$ coated wire, the maximum payload was increased to 1.82 grams, which is around $60 \%$ improvement. The total length of the supporting legs of the robot that were in contact with the water surface was approximately $16 \mathrm{~cm}$. From the surface tension analysis, the theoretical value of the maximum payload was 2.24 grams, which was about $23 \%$ more than the experimental value. The error may be explained by taking into account the bending of the supporting legs, and more rigorous analysis is left for future research. The overall weight of the robot was 0.67 grams. So the Teflon ${ }^{\circledR}$ coated supporting legs can support up to 3 times the total weight of the robot.

Testing maximum payload assured the water strider's static stability after attaching actuators. Actuation experiments followed to prove the feasibility of motion control on the water

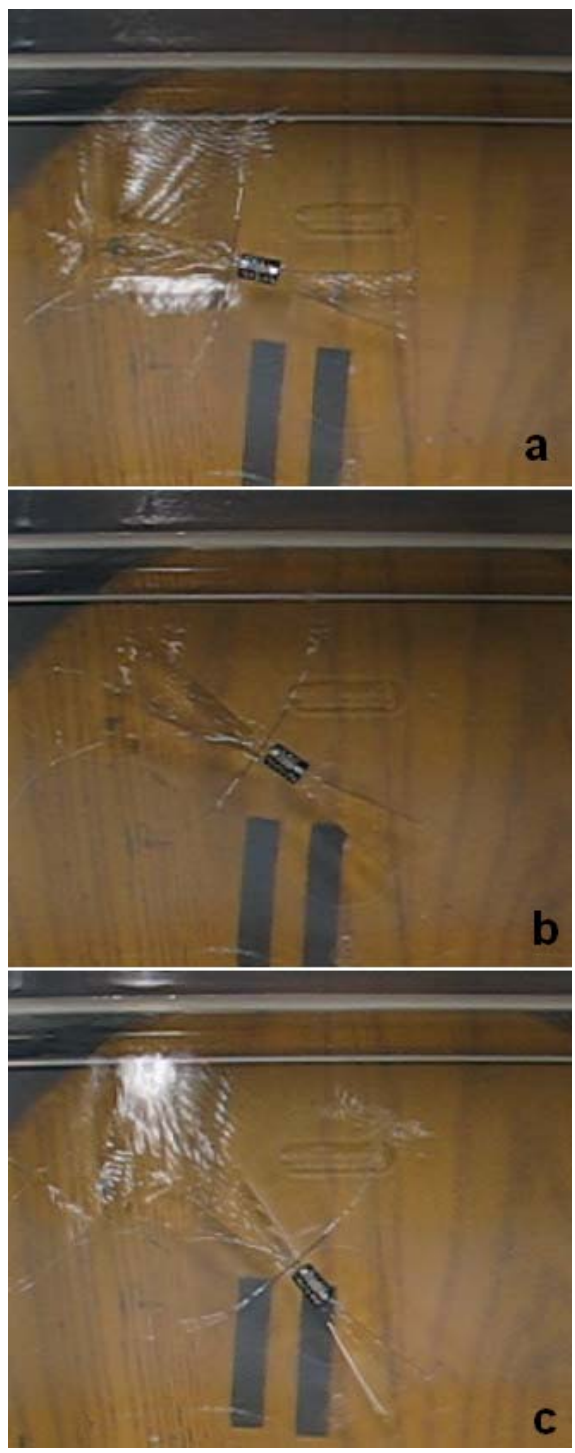

Fig. 15. Right turn in 1.5 second intervals (a-c). 
surface. Two separate sinusoidal input signals with a $90^{\circ}$ phase shift were generated to create the desired elliptical motion of the right and left actuating legs to drive the robot forward. In order to create significant displacement, these signals were amplified, providing piezoelectric actuators with a unipolar voltage ranging from $0 \mathrm{~V}$ to $-150 \mathrm{~V}$. The first few resonant frequencies of the T-shaped actuator part from the ANSYS ${ }^{\circledR}$ analysis were compared to experimental natural frequencies of the entire system. The second resonant frequency of $36.2 \mathrm{~Hz}$ $\left(\right.$ ANSYS $^{\circledR}$ result was $37.57 \mathrm{~Hz}$ ) showed the greatest deflection of the middle actuator. Driving the robot in this frequency allowed the actuating leg tips to be completely lifted up from the water surface before making the next stroke. The same frequency signal with a phase difference of $90^{\circ}$ was used to drive the right/left actuators. Given an arbitrary initial position, simply changing the input signal to the middle actuator as proposed in Section II.E achieved forward (Fig. 14) and backward movement. The maximum speed of the robot in forward motion was $2.3 \mathrm{~cm} / \mathrm{s}$, which was $1.5 \%$ of the water strider's maximum speed.

When the right and left actuators were given opposite signals, the third mode of vibration was excited and the robot could make turns as expected. Although not as maneuverable as forward and backward motion, left and right turning was also plausible (Fig. 15).

Driving the robot in significantly lower frequencies than the resonance frequencies would have ensured stable control, but the amount of actuation in lower driving frequencies was not enough to drive the robot. Since the higher priority was to produce large enough deflection to actuate the robot, the resonant frequencies were chosen as the driving frequencies.

Observing the locomotion on water, the robot created ripple patterns on the water surface as seen in Fig. 15. Further analysis of these surface waves is left for future research. While conducting experiments safety issues were raised, from dealing with an electrically powered device on water. In order to prevent potential short-circuiting of the system and injuries, it is necessary to develop methods to make the robot waterproof in case of submergence, such as coating the electrical parts, actuators or the whole body with a non-wetting polymer.

\section{CONCLUSIONS}

In this paper, inspired by recent studies on insect water striders, critical features that allow them to effectively maneuver on the water surface were implemented, demonstrating a new possible robotic locomotion method on water. Design analysis for the leg and water-surface interface and robot kinematics and dynamics were demonstrated. The actual robot, which was driven in one of its resonant modes, could move forward and backward at the speed of $2.3 \mathrm{~cm} / \mathrm{s}$ and rotate to the right or left without breaking the water surface. However, whether the water strider robot in motion creates hemispherical vortices is not yet verified [7]. The ripples on the water that were created when the robot was in motion are also of interest, which is left for future research. Comparison between boat design and this prototype regarding the efficiency (drag, power, speed, maneuverability, etc.) would clarify the competitiveness of the proposed design. In the near future this robot would become untethered for autonomous and teleoperated control. Attaching miniature biochemical sensors would enable it to be used in monitoring chemicals on water surfaces for environmental monitoring and cleaning applications.

\section{ACKNOWLEDGMENT}

The authors thank Kwon Joong Son for his help on the piezoelectric actuator analysis, Eugene Cheung for his help on the revision of this paper, and all the other members of the NanoRobotics Laboratory for their support and suggestions on this project.

\section{REFERENCES}

[1] J.E. Clark, J.G Cham, S.A. Bailey, E.M. Froehlich, P.K. Nahata, R.J. Full, and M.R. Cutkosky, "Biomimetic design and fabrication of a hexapedal running robot," Proceedings of IEEE International Conference on Robotics and Automation, vol. 4, pp 3643-3649, 2001.

[2] N. Kagawa and H. Kazerooni, "Biomimetic Small Walking Machine," Proceedings of the IEEE/ASME International Conference on Advanced Intelligent Mechatronics, pp. 971-979, 2001.

[3] C. Menon, M. Murphy, and M. Sitti, "Gecko Inspired Surface Climbing Robots," Proceedings of the IEEE International Conference on Robotics and Biomimetics (ROBIO), Shenyang, China, 2004.

[4] F. Chiu, J. Guo, J. Chen, and Y. Lin, "Dynamic characteristic of a biomimetic underwater vehicle;" Proceedings of the IEEE International Symposium on Underwater Technology, pp 172-177, 2002.

[5] S. Guo, Y. Hasegaw, T. Fukuda, and K. Asaka, "Fish-like Underwater Microrobot with Multi DOF," Proceedings of IEEE International Symposium on Micromechatronics and Human Science, pp. 63-68, 2001.

[6] X. Gao and L. Jiang, "Water-repellent legs of water striders," Nature, pp 36, vol. 432, 2004.

[7] D.L. Hu, B. Chan, and J.W.M. Bush, "The hydrodynamics of water strider locomotion," Nature, vol. 424, pp. 663-666, 2003.

[8] M. Sitti, D. Campolo, J. Yan, R.S. Fearing, T. Su, D. Taylor, and T. Sands, "Development of PZT/PZN-PT Unimorph Actuators for Micromechanical Flapping Mechanisms," Proc. of the IEEE Robotics and Automation Conference, pp. 3839-3846, Korea, May 2001.

[9] J.B. Keller, "Surface tension force on a partly submerged body," Physics of Fluids, vol. 10, pp. 3009-3010, 1998.

[10] M. Sitti, "Piezoelectrically actuated four-bar mechanism with two flexible links for micromechanical flying insect thorax," IEEE/ASME Trans. on Mechatronics, vol. 8, no. 1, pp. 26-36, March 2003.

[11] M.S. Weinberg, "Working Equations for Piezoelectric Actuators and Sensors," ASME/IEEE Journal of MEMS, vol. 8, No. 4, 1999. 